Review Article

\title{
Chemical and Immunological Characteristics of Aluminum- Based, Oil-Water Emulsion, and Bacterial-Origin Adjuvants
}

\author{
Susana Martiñón (D, ${ }^{1,2}$ Angel Cisneros, ${ }^{1}$ Sergio Villicaña (iD, 1 \\ Ricardo Hernández-Miramontes, ${ }^{1}$ Edgar Mixcoha, ${ }^{3}$ and Psyché Calderón-Vargas $\mathbb{D}^{4}$ \\ ${ }^{1}$ Neuropharmacology, Biotechnology and Experimental Therapeutic Anti-Addictions Laboratory, National Institute of Psychiatry \\ Ramón de la Fuente Muñiz, Mexico City, Mexico \\ ${ }^{2}$ Faculty of Health Sciences, Anahuac University, Huixquilucan, Estado de Mexico, Mexico \\ ${ }^{3}$ CONACYT Researcher Fellowship-National Institute of Psychiatry Ramón de la Fuente Muñiz, Mexico \\ ${ }^{4}$ Centro de la Conducta S.C., Tijuana, Baja California, Mexico
}

Correspondence should be addressed to Susana Martiñón; susimar2000@yahoo.com

Received 17 December 2018; Revised 2 April 2019; Accepted 15 April 2019; Published 8 May 2019

Academic Editor: Peirong Jiao

Copyright (c) 2019 Susana Martiñón et al. This is an open access article distributed under the Creative Commons Attribution License, which permits unrestricted use, distribution, and reproduction in any medium, provided the original work is properly cited.

\begin{abstract}
Adjuvants are a diverse family of substances whose main objective is to increase the strength, quality, and duration of the immune response caused by vaccines. The most commonly used adjuvants are aluminum-based, oil-water emulsion, and bacterial-origin adjuvants. In this paper, we will discuss how the election of adjuvants is important for the adjuvant-mediated induction of immunity for different types of vaccines. Aluminum-based adjuvants are the most commonly used, the safest, and have the best efficacy, due to the triggering of a strong humoral response, albeit generating a weak induction of cell-mediated immune response. Freund's adjuvant is the most widely used oil-water emulsion adjuvant in animal trials; it stimulates inflammation and causes aggregation and precipitation of soluble protein antigens that facilitate the uptake by antigen-presenting cells (APCs). Adjuvants of bacterial origin, such as flagellin, E. coli membranes, and monophosphoryl lipid A (MLA), are known to potentiate immune responses, but their safety and risks are the main concern of their clinical use. This minireview summarizes the mechanisms that classic and novel adjuvants produce to stimulate immune responses.
\end{abstract}

\section{Introduction}

Vaccines constitute one of the greatest achievements in the history of medicine, their main objective being the prevention of diseases by inducing the immune response. Purified antigen-based vaccines, either synthetic or recombinant, are more specific but less immunogenic than original vaccines formed by live attenuated or inactivated microbes; therefore, associated agents called "adjuvants" are required, which increase their immune response strength, quality, and duration (memory) [1].

Adjuvants aim, ideally, to increase and improve the immunogenicity of antigens by decreasing the amount and number of immunizations; thus, the search for new substances with adjuvant/immunopotentiating activity has been one of the main trends in immunological research for over a decade [2]. The rational design of vaccines involves the logical choice of the immunopotentiator, based on their mode of action and its expected effect on the efficacy and safety of the vaccine [3].

In this paper, we address the use of the most frequently used adjuvants in experimental and clinical trials, describing their immunological characteristics, with the objective of helping the decision on the best adjuvant for each vaccine.

\section{Adjuvants}

One of the most important issues when designing efficient and safe vaccines is the selection of an appropriate adjuvant that meets both the desired immunogenic potential and the 
safety requirements for human use. The best adjuvant is considered to be the one that elicits the most potent immune response while posing the least risk to the individual's health.

Adjuvants, or immunopotentiating agents, are a group of substances with divergent chemical structures that are used to increase, improve, or extend the immune response against a simultaneously administered antigen $[2,4]$. The concept was pioneered in the 1920s by Ramon [5-7] who pointed out that horses that developed an abscess at the diphteria toxoid inoculation site produced higher specific antibody titers than those who did not.

Adjuvants are normally used with several purposes: (a) to improve the immunogenicity of highly purified or recombinant antigens; (b) to reduce the amount of antigen or the number of vaccine administrations required for the development of immunity; (c) to improve the efficiency of vaccines in newborns, the aged, and immunocompromised individuals; and (d) to be used as systems for the delivery of the antigen and its assimilation in mucosa [5].

The most used adjuvants in clinical and experimental trials are enunciated in Figure 1, and the chemical and immunological characteristics of each one are described as follows.

\section{Aluminum-Based Adjuvants}

Aluminum-based adjuvants are used in at least 146 approved vaccines for the prevention of disease, which make them the most commonly used [8]. As a matter of fact, until 1997, aluminum-based adjuvants were the only ones approved for use in humans and remain to be the most stable, safe, tolerated, and effective. The most used aluminum-based adjuvants in vaccines are salts of three types: aluminum hydroxide, aluminum phosphate, and potassium aluminum sulfate [9].

Aluminum hydroxide gels are slightly crystalline and amorphous and have a mineral structure of pseudoboehmite, oxy-based aluminum hydroxide, and $\mathrm{AlO}(\mathrm{OH})$. $\mathrm{nH}_{2} \mathrm{O}$. Gels comprise both micro- and nanoparticles, formed by aggregates of primary crystals of up to 10 $\mathrm{nm}$ in length [10]. Oxyaluminum hydroxide has a surface area of $500 \mathrm{~m}^{2} / \mathrm{g}$, and only the outer layer is antigenassociated by surface adsorption; the association is carried out mainly by electrostatic forces, hydrophobic interactions, hydrogen bonds, ligand exchange, and Van der Waals forces. The point of zero charge (PZC) is 11.4, so in a neutral $\mathrm{pH}$, it is positively charged, allowing antigens to bind primarily to their negative charges. Additionally, either PZC or antigen binding can be altered in both power and stability, combining it with phosphate counterions [8-10].

The mechanisms of action of aluminum hydroxide and, in general, aluminum-based adjuvants include (1) aggregate formation enabling continuous release of antigens; (2) formation of particle structures that promote phagocytosis of antigens by antigen-presenting cells (APC); and (3) induction of local inflammation via the NLRP3 inflammasome, which results in the recruitment and activation of macrophages and increase in the expression of molecules of major histocompatibility complex (MHC) class II and antigen presentation [11]. The activation of the inflammasome induces the secretion of mature IL- $1 \beta$ and IL-18 by dendritic cells and the differentiation of $\mathrm{TH} 2$ cells, promoting the activation of $\mathrm{B}$ cells and the subsequent production of antibodies, predominantly IgG [12, 13] [14]. However, NLRP3-independent antibody production pathways have been shown, as well as a nonphagocytic way of acting of the aluminum hydroxide [13].

Summarizing, aluminum-based adjuvants trigger a strong humoral immune response primarily mediated by secreting antibodies specific to antigens, particularly IgG1, albeit generating a weak induction of cell-mediated immune response [14].

\section{Oil-Water Emulsion Adjuvants}

4.1. Freund's Adjuvant. The most widely used oil-water emulsion adjuvant in animal experimentation is Freund's adjuvant, from which there are two variants: the incomplete (Incomplete Freund's Adjuvant (IFA)) and the complete (Complete Freund's Adjuvant (CFA)) adjuvant [15]. For enhancing the immune response, the CFA contain killed mycobacteria (Mycobacterium tuberculosis) which are responsible for attracting macrophages and other cells to the site of injection, and due to that, it is usually applied in the initial immunizations. Due to its toxicity and secondary reactions, the use of CFA in humans is ineligible as a proper adjuvant; however, IFA is less toxic and therefore suitable for its clinical usage [16].

The CFA is used to prepare oil-water emulsion adjuvants with the immunogen so that the antigen is slowly released and produces a high and long duration stimulation of the immune response. A typical composition of CFA comprises $1 \mathrm{mg}$ Mycobacterium tuberculosis heat killed and dried with $0.85 \mathrm{~mL}$ paraffin and $0.15 \mathrm{~mL}$ of Arlacel ${ }^{\circledR} 83$ (a mixture of oleic, palmitic, stearic, and linoleic esters with 2-(3,4 dihydroxytetrahydrofuranyl)-ethylene glycol) [17]. The $M$. tuberculosis immune effects will be reviewed in Adjuvants of Bacterial Origin.

Common pathways of antigen and adjuvant emulsion inoculation are intradermal, subcutaneous, and intramuscular, although the intraperitoneal pathway is also used [18]. It has the great disadvantage such that its use is restricted only for laboratory animals because it contains mineral oil that is not metabolized by humans and the mycobacterial elements can lead to granulomatous reactions [4].

4.2. Squalene. Squalene is a terpene found in plants and the liver of some animal species, including humans. It acts as a precursor for cholesterol, steroid hormones, and vitamin D. Squalene for commercial purposes is usually extracted from shark liver oil, but it can also be obtained from vegetable oils, such as olive oil and palm oil $[19,20]$. There were reports by Asa et al. [21] that squalene-based vaccines could lead to the production of anti-squalene antibodies [22, 23], although these claims were later criticized and have been a controversial subject $[22,24,25]$. Nevertheless, more than 20 million doses of squalene-based vaccines have been administered 


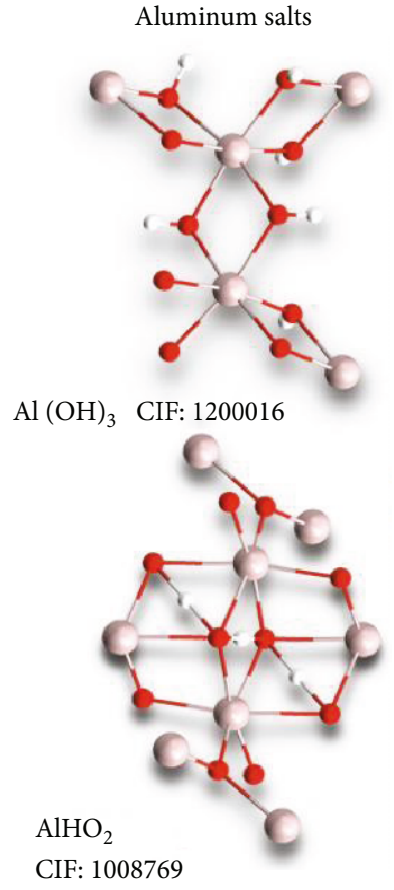

(a)

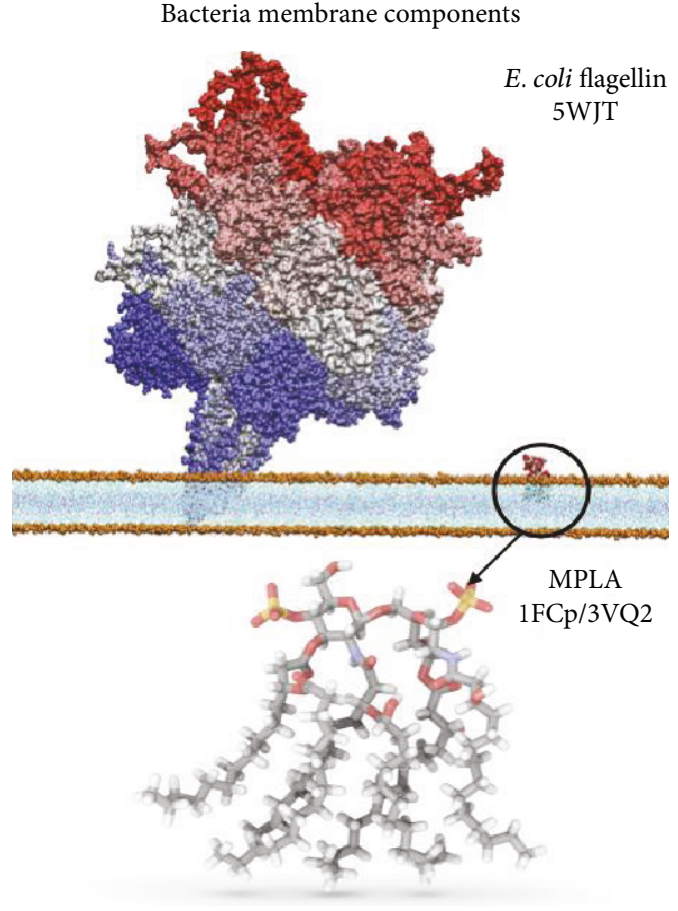

(b)
Complete Freund's adjuvant

Emulsion of Mycobacterium tuberculosis in paraffin oil and mannide monooleate

(c)

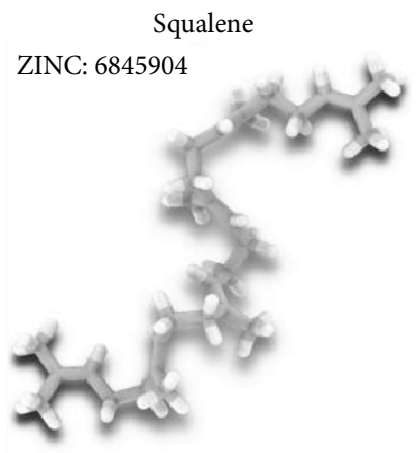

(d)

FIGURE 1: Three-dimensional representation of adjuvants. (a) Crystal structures of aluminum salts used as adjuvants in human vaccines. $\mathrm{Al}(\mathrm{OH})_{3}$ is the most widely used adjuvant in some crystal structures (such as gibbsite) and amorphous forms [86]. Another aluminum salt used in vaccines is aluminum oxide hydroxide such as goethite [87]. (b) Several bacterial membrane proteins are used as adjuvants in order to activate human immune cells. Bacterial flagellin is detected by TLR5 in innate cells activating a high immune response; recently, the B. subtilis flagellin structure was solved using cryomicroscopy under the 5WJT PDB code [88]. On the other hand, phospholipids and lipidic components in the bacterial membrane are recognized as dangerous and activate immune response. (c) The most used adjuvant in animal immunization is an emulsion of oil, paraffin, and M. tuberculosis death cells. (d) Squalene is an oil compound present in the liver of sharks as a precursor of cholesterol metabolism. In recent years, squalene has been accepted as an adjuvant for human vaccination. Immunological results of squalene have demonstrated it to be an efficient adjuvant. The coordinates of squalene were taken from the ZINC15 data bank [89].

worldwide with biosafety results ranging from acceptable to excellent $[25,26]$.

Several adjuvants containing squalene have been used in licensed human vaccines, such as MF59, which is an oil-inwater nanoemulsion containing squalene, and Tween 80 and Span 85 (both surfactants); AS03 and AS04, containing $\alpha$-tocopherol (a form of vitamin $\mathrm{E}$ ); and polysorbate 80 [27]. Squalene is used as an adjuvant for several different vaccines along with antigens from the influenza virus, hepatitis B and $C$ viruses, the herpes simplex virus, etc. [25]. MF59 has been associated with an increase in the activity of helper $\mathrm{T}$ lymphocytes and higher concentrations of immunoglobulins, specifically the IgG1 and IgG2a isotypes. MF59 has also been reported as capable of inducing Th1 responses in $\mathrm{CD}^{+} \mathrm{T}$ lymphocytes [26].

Recent studies have shown that the MF59 adjuvant contributed to seroconversion and seroprotection 21 days after the first administration of an influenza A H1N1 vaccine. The same vaccine elicited higher percentages of seroprotection and seroconversion after 21 and 42 days when adjuvanted than when nonadjuvanted. Regarding the safety of the vaccine, this cohort reported mild to moderate adverse effects, including pain and bruising at the injection site, as well as muscular ache. Nevertheless, none of these adverse reactions lasted beyond 72 hours [28].

4.3. Other Squalene-Based Adjuvants. Formulations including squalene and other compounds have also been tested. GLA-SE is an oil-in-water emulsion with squalene and glucopyranosyl lipid adjuvant (GLA). This formulation has been shown to induce strong signaling through the TLR-4, caspase, IL-18, and IFN- $\gamma$ pathways, leading to a Th1 response [29]. GLA-SE has been used as the adjuvant for a tuberculosis vaccine in humans with potent antibody responses peaking after the second immunization and mild side effects including headaches and fatigue [30]. MPL-SE is a mix of MPL-A (a nontoxic derivative of the lipopolysaccharide of Salmonella minnesota; see Monophosphoryl Lipid A (MPL-A)) with squalene oil, excipients, and water. As it represents an excellent promoter for Th1 responses, there is ongoing research regarding its applicability for leishmaniasis vaccines [26]. Syntex Adjuvant Formulation (SAF) 
is an oil-in-water emulsion that contains squalene, Tween TM 80, and Pluronic TM L121 in phosphate-buffered saline. It is currently in preclinical tests for vaccines containing antigens from the influenza virus, the Epstein-Barr virus, and the Human Immunodeficiency Virus (HIV) [26].

\section{Adjuvants of Bacterial Origin}

5.1. Flagellin. Flagellin is a protein composed of 494 amino acids and is the most important structural protein in the flagella of Gram-negative bacteria [31]. It is composed of three domains (D1, D2, and D3), with D1 and D2 being highly conserved and D3 being hypervariable [32]. As the primary component of flagella, it contributes to motility of bacterial cells. Most of the bacterial flagellin molecules stay in their flagella, but some of them are released to the medium, enabling its recognition by the immune system.

It has been reported that the hyperconserved $\mathrm{N}$ and $\mathrm{C}$ termini of flagellin can be recognized by Toll-like receptor (TLR) molecules, particularly by TLR-5 [33-35]. This ability of flagellin to stimulate TLR-5 makes it an interesting option as an adjuvant. Flagellin concentrations in the range of 1 to $10 \mathrm{nM}$ elicit the maximal intensity of TLR-5 signaling. TLR-5 is expressed on different kinds of cells, which include monocytes, macrophages, neutrophils, lymphocytes, NK cells, and dendritic cells. Recognition of TLR-5 with flagellin leads to signaling via both MyD88-dependent and MyD88independent pathways. These pathways lead to the induction of transcription factors AP-1, NF- $\kappa \mathrm{B}$, and IRF3 [32]. These, in turn, trigger the production of cytokines and chemokines that recruit dendritic cells, T lymphocytes, and B lymphocytes to lymph nodes [31]. Flagellin can also promote strong Ag-specific CD $4^{+}$T-cell responses by interacting with TLR-5 on CD11 ${ }^{+}$cells. As this results in high antibody titers, flagellin exhibits potential as an adjuvant $[36,37]$.

Flagellin has been used as an adjuvant by joint administration with the main antigen and through fusion proteins resulting from the addition of epitopes linked to the flagellin molecule. Several studies have been made in order to determine in which regions of the flagellin molecule the epitopes should be inserted to maximize antibody titers, but no definitive conclusions have been reached. Song et al. obtained optimal antibody titers by introducing a hemagglutinin epitope in the hypervariable region of flagellin [38]. Other studies reported that inserting L1R epitopes in the hypervariable region does not produce antibodies [31] and that inserting L1R epitopes in the N-terminus of flagellin could lead to antibodies that interact with the native L1R [39]. Alternatively, Lin et al. showed that insertion of epitopes towards the C-terminus of flagellin can induce signaling via NLRC4 and NAIP5, leading to $\mathrm{CD} 8^{+} \mathrm{T}$-cell responses against tumor cells [40]. This variety of responses exhibits the versatility of flagellin as an adjuvant for different purposes depending on the region of antigen insertion, but further studies are required to fully harness this potential.

The use of flagellin as an adjuvant has several advantages regarding safety: only low doses are required for it to be effective, it does not elicit the synthesis of IgE, no toxicity has been associated with its intranasal administration in animal models, and it can be easily produced in large quantities. Nevertheless, studies in humans are still in phase I and have not been conclusive regarding the adverse effects that could be derived from its use [31]. A study involving flagellin fusion proteins for an influenza A H1N1 vaccine reported that some individuals showed systemic adverse effects. However, some of them were stabilized after 4 days of rest, and another one did so after taking nonsteroidal anti-inflammatory drugs (NSAIDs) [41].

5.2. Bacterial Membranes. E. coli is the most widely studied Gram-negative prokaryotic microorganism in numerous areas of science. The structure of its membrane, as shown in Figure 1, has several relevant features that can be used in the field of immunology and the design of vaccines and adjuvants. As it is a Gram-negative bacterium, its cell wall has an inner layer that is composed of peptidoglycan that comprises only $10 \%$ of the whole structure, whereas most of the cell wall is formed by an outer membrane. The outer membrane is composed of phospholipids and proteins, just like the cytoplasmic membrane, as well as polysaccharides. Lipids and polysaccharides in the outer membrane are usually bound and form a complex called lipopolysaccharide (LPS), which is toxic for animals. The polysaccharides in LPS and polysaccharide $\mathrm{O}$ comprise its core, whereas its lipid component is known as lipid A [42]. A noteworthy feature of Gramnegative bacteria is the release of outer membrane vesicles (OMVs). OMVs are spherical, nanometric vesicles that are released during normal growth and are formed by protuberances in the outer membrane, so they contain LPS, peptidoglycans, phospholipids, and proteins [43, 44].

OMVs represent a novel approach for the development of vaccine adjuvants because of their inherent inflammatory potential, as they stimulate the immune innate system. They activate simultaneously the humoral response and both $\mathrm{CD}^{+} \mathrm{T}$-cell and B-cell responses [43-45]. This mechanism is driven primarily by recognition of LPS and other molecules on membranes by TLR molecules and the complement system [44, 46, 47], leading to the recruitment of antigenpresenting cells [48]. The joint stimulation by the molecules present in OMVs results in a more potent response than that elicited by LPS on its own [49, 50], which makes OMVs attractive as adjuvants. Another immunogenic molecule present in bacterial membranes is protein $\mathrm{D}$, which has been used as an adjuvant for a vaccine against Haemophilus influenzae [51].

Different approaches have been proposed to use OMVs as adjuvants. These include joint administration [52], adding desired epitopes to proteins displayed on the surface of the OMVs $[53,54]$, and delivering proteins within the OMVs [55]. Antibody titers have been higher for the fusion proteins displayed on the surface of the OMVs [43], suggesting vaccines that expose the target epitope could be more successful.

Several vaccines have been put forward using OMVs as adjuvants. Early examples include vaccines against Neisseria meningitidis, which have shown an effectiveness of $73 \%$ or higher in different cohorts [56-58]. These vaccines have been used to fight epidemics and have been part of vaccination programs for more than 20 years $[59,60]$. There are some 
other vaccines that use OMVs and have started moving to clinical trials because of their safety and effectiveness. These include vaccines against allergens (phase I clinical trials) [61, 62], Shigella flexneri (phase I and II clinical trials) [63, 64], and influenza [65]. These vaccines have been administered intranasally and have elicited antibody production with very minor side effects, which highlights the potential of OMVs as adjuvants. Moreover, immunostimulatory proteins and LPS from OMVs do not replicate, which increase their safety $[43,44]$.

Nevertheless, the use of OMVs as adjuvants poses several challenges regarding production and design. Their mass production would be a complex procedure since their content of some endotoxins must be monitored to avoid excess inflammation [66-68]. In particular, the dose of LPS must be controlled accurately because OMVs with low LPS are less-effective adjuvants, while excessive LPS can cause toxic effects $[69,70]$.

5.3. Monophosphoryl Lipid A (MPL-A). LPS is composed of three different regions, namely, lipid $\mathrm{A}$, the core, and a specific glycan. Lipid A is noteworthy because it is responsible for anchoring LPS to the outer membrane and for the endotoxic activity of LPS [71]. Toxicity of lipid A is elicited by the potent stimulation of TLR-4 and intracellular signaling that activates caspases $[72,73]$. The toxic capability of lipid A can be diminished by means of some structural changes, such as removal of the C1-glucosamine phosphate group, which yields monophosphoryl lipid A (MPL-A) [8]. MPL-A has been shown to induce maturation of dendritic cells, $\mathrm{CD} 4^{+}$ T-cell clonal expansion, and Th1 responses without the inflammatory effects of LPS $[68,74]$. However, $\mathrm{CD}^{+} \mathrm{T}$-cell clonal expansion and Th1 differentiation induced by MPL$\mathrm{A}$ is not as long-lasting as that induced by LPS, as T-cell counts induced by MPL-A are lower than those induced by LPS after 21 days [68]. Other studies have shown that MPL-A induces JNK- and mTOR-dependent signaling in macrophages and dendritic cells. This pathway leads to increases in the metabolic activity of macrophages for antimicrobial purposes [75] and the production of proinflammatory cytokines in dendritic cells [76].

MPL-A obtained from Salmonella minnesota was historically the first TLR ligand to be approved for use in humans as an adjuvant [26]. However, MPL-A by itself is not water soluble, which has led to the development of different vehicles to increase its bioavailability after administration. Some strategies that have been explored are the adsorption of MPL-A by aluminum hydroxide molecules and the delivery of MPL-A in liposomes [8]. Both formulations offer advantages. The formulation of MPL-A adsorbed by aluminum hydroxide (ASO4) elicits a higher antibody response with fewer doses than aluminum hydroxide by itself [77]. In turn, liposomes have been successful as adjuvants of vaccines that use immunogenic carrier proteins to induce an immune response against a hapten, that is, a molecule that normally would not induce an immune response [78] and DNA vaccines [79].

Clinical trials for vaccines with MPL-A have been successful. A vaccine for human papilloma virus (HPV) elicited high antibody titers and had only injection site reactions as adverse effects. Antibody titers were particularly high for adolescent girls, suggesting there is an ideal age for administration of the vaccine [77]. Another study showed that the use of liposomes containing MPL-A and adsorbed by aluminum hydroxide could elicit immune responses against repeat-based malaria antigens [80]. However, a potential drawback of the large-scale production of MPL-A for vaccines is that it is obtained through extensive processing of LPS. This leads to large variability between batches and could compromise the efficiency of vaccines [81].

5.3.1. Mycobacterium Tuberculosis. In Oil-Water Emulsion Adjuvants, the CFA was mentioned; however, since it is complemented with Mycobacterium tuberculosis, its principal mechanism of action must be mentioned in this section.

The active components conferred by mycobacteria are a dipeptide, N-acetylmuramyl-L-alanine-D-isoglutamine (MDP), a molecule that activates macrophages and dendritic cells through the nucleotide-binding oligomerization domain containing 2 (NOD2) and skeletal elements of the bacterial cell wall $[82,83]$. Besides stimulating inflammation, adjuvants cause aggregation and precipitation of soluble protein antigens to form particles that facilitate their efficient uptake by APCs. The particulate nature of the antigen also reduces the speed with which the antigen is removed from the system, and this action favors the inflammasome activation [84]. The CFA promotes Th1 subpopulation, promotes synthesis of $\operatorname{IgG}$ rather than IgM, inhibits the induction of tolerance, and favors delayed hypersensitivity reactions [85].

\section{Discussion}

The selection of the "best adjuvant" is relative to the goal of the use; i.e., it will be the one that helps to develop an immune response according to the needs of the antigen of interest. As discussed above, some objectives require the robust production of antibodies $[25,28,38,61,63-$ $65,78]$. In such cases, adjuvants such as squalene or even flagellin help the fusion of proteins with the epitope of interest in the hypervariable region OMV, and liposomes carrying MPL-A have been primarily explored because of the immune responses they elicit. Alternatively, other cases $[26,40]$ require a cytotoxic T-cell response, which is better elicited by epitopes of interest inserted at the C-terminus of flagellin or adjuvants like squalene oil-inwater emulsions. Thus, adjuvant selection is a critical step in vaccine or immunotherapy design.

Equally important, the adjuvant needs to be safe and should have the least intense adverse reactions or, preferably, that it does not have them. Successful adjuvants for vaccines should be easy to access with low cost, as to guarantee that they can be used in any final population that requires the vaccine to be developed. Finally, it is desirable that the adjuvant is applied only once. This review offers chemical and immunological characteristics of the most popular adjuvants. However, it is a very broad area of research, which requires 
more studies and the invention of new pharmacological formulations, either combinations of adjuvants already in use or of new molecules.

\section{Conclusion}

Adjuvants are powerful elements that help with the development of robust immune responses to vaccines. The selection of an adjuvant for each type of vaccine must be made by clearly defining its objective. This simple choice can and will favor the best choice to improve the functionality of future vaccines against numerous diseases.

\section{Conflicts of Interest}

The authors declare that there are no conflicts of interest regarding the publication of this paper.

\section{Authors' Contributions}

Angel Cisneros and Sergio Villicaña contributed equally to this work.

\section{Acknowledgments}

We thank the Instituto Nacional de Psiquiatría Ramón de la Fuente Muñiz, especially Dr. Benito Anton (RIP) and Dr. Alberto Salazar; the Faculty of Health Sciences of Anáhuac University; and CONACYT.

\section{References}

[1] A. Batista-Duharte, M. Lastre, and O. Pérez, "Immunological adjuvants. Determinant factors in the efficacy-toxicity ratio of the contemporary vaccines," Enfermedades Infecciosas y Microbiología Clínica, vol. 32, no. 2, pp. 106-114, 2014.

[2] S. G. Reed, M. T. Orr, and C. B. Fox, "Key roles of adjuvants in modern vaccines," Nature Medicine, vol. 19, no. 12, pp. 15971608, 2013.

[3] E. De Gregorio and R. Rappuoli, "From empiricism to rational design: a personal perspective of the evolution of vaccine development," Nature Reviews Immunology, vol. 14, no. 7, pp. 505-514, 2014.

[4] G. Sierra and B. Tamargo, "Adyuvantes inmunológicos para vacunas humanas: estado actual, tendencias mundiales y en Cuba," Revista Anales de La Academia de Ciencias de Cuba, vol. 1, no. 2, p. 1, 2011.

[5] N. Petrovsky and J. C. Aguilar, "Vaccine adjuvants: current state and future trends," Immunology and Cell Biology, vol. 82, no. 5, pp. 488-496, 2004.

[6] G. Ramon, "Sur l'augmentation anormale de l'antitoxine chez les chevaux producteurs de serum antidiphterique," Bulletin de la Société Centrale de Médecine Vétérinaire, vol. 101, pp. 227234, 1925.

[7] G. Ramon, "Procedes Pour Acroitre La Production Des Antitoxins," Annales de l'Institut Pasteur, vol. 40, pp. 110, 1926.

[8] C. R. Alving, G. R. Matyas, O. Torres, R. Jalah, and Z. Beck, "Adjuvants for vaccines to drugs of abuse and addiction," Vaccine, vol. 32, no. 42, pp. 5382-5389, 2014.
[9] L. A. Brito, P. Malyala, and D. T. O’Hagan, "Vaccine adjuvant formulations: a pharmaceutical perspective," Seminars in Immunology, vol. 25, no. 2, pp. 130-145, 2013.

[10] B. S. Powell, A. K. Andrianov, and P. C. Fusco, "Polyionic vaccine adjuvants: another look at aluminum salts and polyelectrolytes," Clinical and Experimental Vaccine Research, vol. 4, no. 1, pp. 23-45, 2015.

[11] E.-J. Ko, Y.-T. Lee, K.-H. Kim et al., "Roles of aluminum hydroxide and monophosphoryl lipid A adjuvants in overcoming CD4 $\mathrm{T}$ cell deficiency to induce isotype-switched IgG antibody responses and protection by T-dependent influenza vaccine," The Journal of Immunology, vol. 198, no. 1, pp. 279-291, 2017.

[12] N. I. Ho, L. G. M. Huis in 't Veld, T. K. Raaijmakers, and G. J. Adema, "Adjuvants enhancing cross-presentation by dendritic cells: the key to more effective vaccines?," Frontiers in Immunology, vol. 9, 2018.

[13] E. Shardlow, M. Mold, and C. Exley, "Unraveling the enigma: elucidating the relationship between the physicochemical properties of aluminium-based adjuvants and their immunological mechanisms of action," Allergy, Asthma \& Clinical Immunology, vol. 14, no. 1, 2018.

[14] E. Oleszycka and E. C. Lavelle, "Immunomodulatory properties of the vaccine adjuvant alum," Current Opinion in Immunology, vol. 28, pp. 1-5, 2014.

[15] M. A. Behr and M. Divangahi, "Freund's adjuvant, NOD2 and mycobacteria," Current Opinion in Microbiology, vol. 23, pp. 126-132, 2015.

[16] A. Malik, M. Gupta, V. Gupta, H. Gogoi, and R. Bhatnagar, "Novel application of trimethyl chitosan as an adjuvant in vaccine delivery," International Journal of Nanomedicine, vol. 13, pp. 7959-7970, 2018.

[17] P. Pellegrino, E. Clementi, and S. Radice, "On vaccine's adjuvants and autoimmunity: current evidence and future perspectives," Autoimmunity Reviews, vol. 14, no. 10, pp. 880-888, 2015.

[18] P. V. Beirne, S. Hennessy, S. L. Cadogan, F. Shiely, T. Fitzgerald, and F. MacLeod, "Needle size for vaccination procedures in children and adolescents," Cochrane Database of Systematic Reviews, no. 6, article CD010720, 2015.

[19] R. Ambra, F. Natella, S. Lucchetti, V. Forte, and G. Pastore, " $\alpha$ Tocopherol, $\beta$-carotene, lutein, squalene and secoiridoids in seven monocultivar Italian extra-virgin olive oils," International Journal of Food Sciences and Nutrition, vol. 68, no. 5, pp. 538-545, 2017.

[20] G. Lippi, G. Targher, and M. Franchini, "Vaccination, squalene and anti-squalene antibodies: facts or fiction?," European Journal of Internal Medicine, vol. 21, no. 2, pp. 70-73, 2010.

[21] P. B. Asa, Y. Cao, and R. F. Garry, "Antibodies to squalene in Gulf War syndrome," Experimental and Molecular Pathology, vol. 68, no. 1, pp. 55-64, 2000.

[22] P. B. Asa, Y. Cao, and R. F. Garry, "Reply," Experimental and Molecular Pathology, vol. 68, no. 3, pp. 197-198, 2000.

[23] P. B. Asa, R. B. Wilson, and R. F. Garry, "Antibodies to squalene in recipients of anthrax vaccine," Experimental and Molecular Pathology, vol. 73, no. 1, pp. 19-27, 2002.

[24] C. R. Alving and J. D. Grabenstein, "Letter," Experimental and Molecular Pathology, vol. 68, no. 3, pp. 196-197, 2000.

[25] G. Del Giudice, E. Fragapane, R. Bugarini et al., "Vaccines with the MF59 adjuvant do not stimulate antibody responses 
against squalene," Clinical and Vaccine Immunology, vol. 13, no. 9, pp. 1010-1013, 2006.

[26] S. G. Reed, S. Bertholet, R. N. Coler, and M. Friede, "New horizons in adjuvants for vaccine development," Trends in Immunology, vol. 30, no. 1, pp. 23-32, 2009.

[27] A. M. Harandi, "Systems analysis of human vaccine adjuvants," Seminars in Immunology, vol. 39, pp. 30-34, 2018.

[28] T. W. Clark, M. Pareek, K. Hoschler et al., "Trial of 2009 influenza A (H1N1) monovalent MF59-adjuvanted vaccine," The New England Journal of Medicine, vol. 361, no. 25, pp. 24242435, 2009.

[29] A. L. Desbien, S. J. Reed, H. R. Bailor et al., "Squalene emulsion potentiates the adjuvant activity of the TLR4 agonist, GLA, via inflammatory caspases, IL-18, and IFN- $\gamma$," European Journal of Immunology, vol. 45, no. 2, pp. 407-417, 2015.

[30] R. N. Coler, T. A. Day, R. Ellis et al., "The TLR-4 agonist adjuvant, GLA-SE, improves magnitude and quality of immune responses elicited by the ID93 tuberculosis vaccine: first-in-human trial," NPJ Vaccines, vol. 3, no. 1, p. 34, 2018.

[31] S. B. Mizel and J. T. Bates, "Flagellin as an adjuvant: cellular mechanisms and potential," Journal of Immunology, vol. 185, no. 10, pp. 5677-5682, 2010.

[32] I. A. Hajam, P. A. Dar, I. Shahnawaz, J. C. Jaume, and J. H. Lee, "Bacterial flagellin-a potent immunomodulatory agent," Experimental \& Molecular Medicine, vol. 49, no. 9, article e373, 2017.

[33] T. D. Eaves-Pyles, H. R. Wong, K. Odoms, and R. B. Pyles, "Salmonella flagellin-dependent proinflammatory responses are localized to the conserved amino and carboxyl regions of the protein," Journal of Immunology, vol. 167, no. 12, pp. 7009-7016, 2001.

[34] F. Hayashi, T. K. Means, and A. D. Luster, "Toll-like receptors stimulate human neutrophil function," Blood, vol. 102, no. 7, pp. 2660-2669, 2003.

[35] K. G. K. Murthy, A. Deb, S. Goonesekera, C. Szabó, and A. L. Salzman, "Identification of conserved domains in Salmonella muenchen flagellin that are essential for its ability to activate TLR5 and to induce an inflammatory response in vitro," The Journal of Biological Chemistry, vol. 279, no. 7, pp. 56675675, 2004.

[36] J. T. Bates, S. Uematsu, S. Akira, and S. B. Mizel, "Direct stimulation of $t l r 5^{+/+} \mathrm{CD} 11 \mathrm{c}^{+}$cells is necessary for the adjuvant activity of flagellin," The Journal of Immunology, vol. 182, no. 12, pp. 7539-7547, 2009.

[37] S. J. McSorley, B. D. Ehst, Y. Yu, and A. T. Gewirtz, "Bacterial flagellin is an effective adjuvant for CD4 T cells in vivo," The Journal of Immunology, vol. 169, no. 7, pp. 3914-3919, 2002.

[38] L. Song, Y. Zhang, N. E. Yun et al., "Superior efficacy of a recombinant flagellin:H5N1 HA globular head vaccine is determined by the placement of the globular head within flagellin," Vaccine, vol. 27, no. 42, pp. 5875-5884, 2009.

[39] K. N. Delaney, J. P. Phipps, J. B. Johnson, and S. B. Mizel, “A recombinant flagellin-poxvirus fusion protein vaccine elicits complement-dependent protection against respiratory challenge with vaccinia virus in mice," Viral Immunology, vol. 23, no. 2, pp. 201-210, 2010.

[40] K.-H. Lin, L.-S. Chang, C.-Y. Tian et al., "Carboxyl-terminal fusion of E7 into flagellin shifts TLR5 activation to NLRC4/NAIP5 activation and induces TLR5-independent anti-tumor immunity," Scientific Reports, vol. 6, no. 1, article 24199, 2016.

[41] J. J. Treanor, D. N. Taylor, L. Tussey et al., "Safety and immunogenicity of a recombinant hemagglutinin influenza-flagellin fusion vaccine (VAX125) in healthy young adults," Vaccine, vol. 28, no. 52, pp. 8268-8274, 2010.

[42] M. T. Madigan, J. M. Martinko, P. V. Dunlap, and D. P. Clark, Brock Biology of Microorganisms. Vol. 11, Pearson, 12th edition, 2009.

[43] M. H. Daleke-Schermerhorn, T. Felix, Z. Soprova et al., "Decoration of outer membrane vesicles with multiple antigens by using an autotransporter approach," Applied and Environmental Microbiology, vol. 80, no. 18, pp. 5854-5865, 2014.

[44] D. H. Lee, S.-H. Kim, W. Kang et al., "Adjuvant effect of bacterial outer membrane vesicles with penta-acylated lipopolysaccharide on antigen-specific T cell priming," Vaccine, vol. 29, no. 46, pp. 8293-8301, 2011.

[45] R. C. Alaniz, B. L. Deatherage, J. C. Lara, and B. T. Cookson, "Membrane vesicles are immunogenic facsimiles of Salmonella typhimurium that potently activate dendritic cells, prime B and $\mathrm{T}$ cell responses, and stimulate protective immunity in vivo," The Journal of Immunology, vol. 179, no. 11, pp. 7692-7701, 2007.

[46] B. S. Collins, "Gram-negative outer membrane vesicles in vaccine development," Discovery Medicine, vol. 12, no. 62, pp. 715, 2011.

[47] P. Massari, A. Visintin, J. Gunawardana et al., "Meningococcal porin PorB binds to TLR2 and requires TLR1 for signaling," Journal of Immunology, vol. 176, no. 4, pp. 2373-2380, 2006.

[48] K. Tan, R. Li, X. Huang, and Q. Liu, "Outer membrane vesicles: current status and future direction of these novel vaccine adjuvants," Frontiers in Microbiology, vol. 9, p. 783, 2018.

[49] T. N. Ellis and M. J. Kuehn, "Virulence and immunomodulatory roles of bacterial outer membrane vesicles," Microbiology and Molecular Biology Reviews, vol. 74, no. 1, pp. 81-94, 2010.

[50] S. B. Park, H. B. Jang, S. W. Nho et al., "Outer membrane vesicles as a candidate vaccine against Edwardsiellosis," PLoS One, vol. 6, no. 3, article e17629, 2011.

[51] Q. Su, Y. Yi, F. Qiu et al., "Immune responses to HBsAg conjugated to protein D of non-typeable Haemophilus influenzae in mice," PLoS One, vol. 10, no. 2, article e0117736, 2015.

[52] J. Findlow, S. Taylor, A. Aase et al., "Comparison and correlation of Neisseria meningitidis serogroup B immunologic assay results and human antibody responses following three doses of the Norwegian meningococcal outer membrane vesicle vaccine MenBvac," Infection and Immunity, vol. 74, no. 8, pp. 4557-4565, 2006.

[53] J.-Y. Kim, A. M. Doody, D. J. Chen et al., "Engineered bacterial outer membrane vesicles with enhanced functionality," Journal of Molecular Biology, vol. 380, no. 1, pp. 51-66, 2008.

[54] J. Schroeder and T. Aebischer, "Recombinant outer membrane vesicles to augment antigen-specific live vaccine responses," Vaccine, vol. 27, no. 48, pp. 6748-6754, 2009.

[55] M. Muralinath, M. J. Kuehn, K. L. Roland, and R. Curtiss III, "Immunization with Salmonella enterica serovar Typhimurium-derived outer membrane vesicles delivering the pneumococcal protein PspA confers protection against challenge with Streptococcus pneumoniae," Infection and Immunity, vol. 79, no. 2, pp. 887-894, 2011. 
[56] R. Arnold, Y. Galloway, A. McNicholas, and J. O’Hallahan, "Effectiveness of a vaccination programme for an epidemic of meningococcal B in New Zealand," Vaccine, vol. 29, no. 40, pp. 7100-7106, 2011.

[57] E. Rosenqvist, E. A. Høiby, E. Wedege et al., "Human antibody responses to meningococcal outer membrane antigens after three doses of the Norwegian group B meningococcal vaccine," Infection and Immunity, vol. 63, no. 12, pp. 4642-4652, 1995.

[58] G. V. Sierra, H. C. Campa, N. M. Varcacel et al., "Vaccine against group B Neisseria meningitidis: protection trial and mass vaccination results in Cuba," NIPH Annals, vol. 14, no. 2, pp. 195-207, 1991.

[59] R. Acevedo, S. Fernández, C. Zayas et al., "Bacterial outer membrane vesicles and vaccine applications," Frontiers in Immunology, vol. 5, p. 121, 2014.

[60] J. Holst, P. Oster, R. Arnold et al., "Vaccines against meningococcal serogroup $\mathrm{B}$ disease containing outer membrane vesicles (OMV): lessons from past programs and implications for the future," Human Vaccines \& Immunotherapeutics, vol. 9, no. 6, pp. 1241-1253, 2013.

[61] M. Lastre, O. Pérez, A. Labrada et al., "Bacterial derived proteoliposome for allergy vaccines," Vaccine, vol. 24, Supplement 2, pp. S34-S35, 2006.

[62] National Center of Bioproducts, "Subcutaneous immunotherapy with PROLINEM-Asthma-Adults-Fase I," RPCEC: Registro Público Cubano de Ensayos Clínicos, 2013, http://rpcec.sld .cu/trials/RPCEC00000139-En.

[63] L. F. Fries, A. D. Montemarano, C. P. Mallett, D. N. Taylor, T. L. Hale, and G. H. Lowell, "Safety and immunogenicity of a proteosome-Shigella flexneri 2a lipopolysaccharide vaccine administered intranasally to healthy adults," Infection and Immunity, vol. 69, no. 7, pp. 4545-4553, 2001.

[64] T. Jones, S. Cyr, F. Allard, N. Bellerose, G. H. Lowell, and D. S. Burt, "Protollin: a novel adjuvant for intranasal vaccines," Vaccine, vol. 22, no. 27-28, pp. 3691-3697, 2004.

[65] D. Burt, C. Mallett, M. Plante, J. Zimmermann, K. Torossian, and L. Fries, "Proteosome-adjuvanted intranasal influenza vaccines: advantages, progress and future considerations," Expert Review of Vaccines, vol. 10, no. 3, pp. 365-375, 2011.

[66] C. Arigita, T. Luijkx, W. Jiskoot et al., "Well-defined and potent liposomal meningococcal B vaccines adjuvated with LPS derivatives," Vaccine, vol. 23, no. 43, pp. 5091-5098, 2005.

[67] J. Lucidarme, M. Comanducci, J. Findlow et al., "Characterization of $\mathrm{fHbp}$, Nhba (gna2132), nadA, porA, sequence type (ST), and genomic presence of IS1301 in group B meningococcal ST269 clonal complex isolates from England and Wales," Journal of Clinical Microbiology, vol. 47, no. 11, pp. 35773585, 2009.

[68] B. S. Thompson, P. M. Chilton, J. R. Ward, J. T. Evans, and T. C. Mitchell, "The low-toxicity versions of LPS, MPL ${ }^{\circledR}$ adjuvant and RC529, are efficient adjuvants for $\mathrm{CD}^{+}{ }^{+} \mathrm{T}$ cells," Journal of Leukocyte Biology, vol. 78, no. 6, pp. 1273-1280, 2005.

[69] Y. M. D. Gnopo, H. C. Watkins, T. C. Stevenson, M. P. DeLisa, and D. Putnam, "Designer outer membrane vesicles as immunomodulatory systems - reprogramming bacteria for vaccine delivery," Advanced Drug Delivery Reviews, vol. 114, pp. 132-142, 2017.

[70] A. Nalbantsoy, N. U. Karabay-Yavasoglu, and I. DelilogluGurhan, "Determination of in vivo toxicity and in vitro cytotoxicity of lipopolysaccharide isolated from Salmonella enteritidis and its potential use for production of polyclonal antibody," Food and Agricultural Immunology, vol. 22, no. 3, pp. 271-281, 2011.

[71] A. Molinaro, O. Holst, F. di Lorenzo et al., "Chemistry of lipid A: at the heart of innate immunity," Chemistry, vol. 21, no. 2, pp. 500-519, 2015.

[72] N. Kayagaki, M. T. Wong, I. B. Stowe et al., "Noncanonical inflammasome activation by intracellular LPS independent of TLR4," Science, vol. 341, no. 6151, pp. 1246-1249, 2013.

[73] J. Shi, Y. Zhao, Y. Wang et al., "Inflammatory caspases are innate immune receptors for intracellular LPS," Nature, vol. 514, no. 7521, pp. 187-192, 2014.

[74] J. Ismaili, J. Rennesson, E. Aksoy et al., "Monophosphoryl lipid A activates both human dendritic cells and T cells," The Journal of Immunology, vol. 168, no. 2, pp. 926-932, 2002.

[75] B. A. Fensterheim, J. D. Young, L. Luan et al., “The TLR4 agonist monophosphoryl lipid A drives broad resistance to infection via dynamic reprogramming of macrophage metabolism," Journal of Immunology, vol. 200, no. 11, pp. 3777-3789, 2018.

[76] F. Blanco-Pérez, A. Goretzki, S. Wolfheimer, and S. Schülke, "The vaccine adjuvant MPLA activates glycolytic metabolism in mouse mDC by a JNK-dependent activation of mTOR-signaling," Molecular Immunology, vol. 106, pp. 159-169, 2019.

[77] S. J. Keam and D. M. Harper, "Human papillomavirus types 16 and 18 vaccine (Recombinant, AS04 Adjuvanted Adsorbed) [Cervarix ${ }^{\mathrm{TM}}$ ]," Drugs, vol. 68, no. 3, pp. 359372, 2008.

[78] G. R. Matyas, A. V. Mayorov, K. C. Rice et al., "Liposomes containing monophosphoryl lipid A: a potent adjuvant system for inducing antibodies to heroin hapten analogs," Vaccine, vol. 31, no. 26, pp. 2804-2810, 2013.

[79] M. Tian, Z. Zhou, S. Tan, X. Fan, L. Li, and N. Ullah, "Formulation in DDA-MPLA-TDB liposome enhances the immunogenicity and protective efficacy of a DNA vaccine against mycobacterium tuberculosis infection," Frontiers in Immunology, vol. 9, 2018.

[80] L. F. Fries, D. M. Gordon, R. L. Richards et al., "Liposomal malaria vaccine in humans: a safe and potent adjuvant strategy," Proceedings of the National Academy of Sciences of the United States of America, vol. 89, no. 1, pp. 358362, 1992.

[81] K. A. Gregg, E. Harberts, F. M. Gardner et al., "Rationally designed TLR4 ligands for vaccine adjuvant discovery," mBio, vol. 8, no. 3, 2017.

[82] R. Caruso, N. Warner, N. Inohara, and G. Núñez, "NOD1 and NOD2: signaling, host defense, and inflammatory disease," Immunity, vol. 41, no. 6, pp. 898-908, 2014.

[83] D. M. Prigozhin, D. Mavrici, J. P. Huizar, H. J. Vansell, and T. Alber, "Structural and biochemical analyses of mycobacterium tuberculosis $\mathrm{N}$-acetylmuramyl-L-alanine amidase Rv3717 point to a role in peptidoglycan fragment recycling," The Journal of Biological Chemistry, vol. 288, no. 44, pp. 31549-31555, 2013.

[84] S. Neumann, K. Burkert, R. Kemp, T. Rades, P. Rod Dunbar, and S. Hook, "Activation of the NLRP3 inflammasome is not a feature of all particulate vaccine adjuvants," Immunology and Cell Biology, vol. 92, no. 6, pp. 535-542, 2014.

[85] G. Tadepalli, B. Konduru, H. S. Murali, and H. V. Batra, "Intraperitoneal administration of a novel chimeric immunogen (rOP) elicits IFN- $\gamma$ and IL-12p70 protective immune 
response in BALB/c mice against virulent Brucella," Immunology Letters, vol. 192, pp. 79-87, 2017.

[86] H. Saalfeld and M. Wedde, "Refinement of the crystal structure of gibbsite, $\mathrm{Al}(\mathrm{OH})_{3}$," Zeitschrift Für Kristallographie, vol. 139, no. 1-2, pp. 129-135, 1974.

[87] J.-L. Hazemann, J. F. Bérar, and A. Manceau, "Rietveld studies of the aluminium-iron substitution in synthetic goethite," Materials Science Forum, vol. 79-82, pp. 821-826, 1991.

[88] F. Wang, A. M. Burrage, S. Postel et al., "A structural model of flagellar filament switching across multiple bacterial species," Nature Communications, vol. 8, no. 1, p. 960, 2017.

[89] T. Sterling and J. J. Irwin, "ZINC 15 - ligand discovery for everyone," Journal of Chemical Information and Modeling, vol. 55, no. 11, pp. 2324-2337, 2015. 


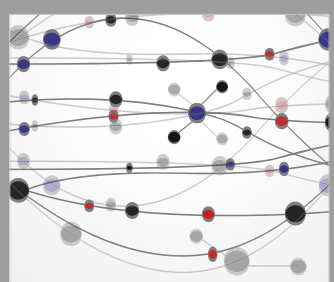

The Scientific World Journal
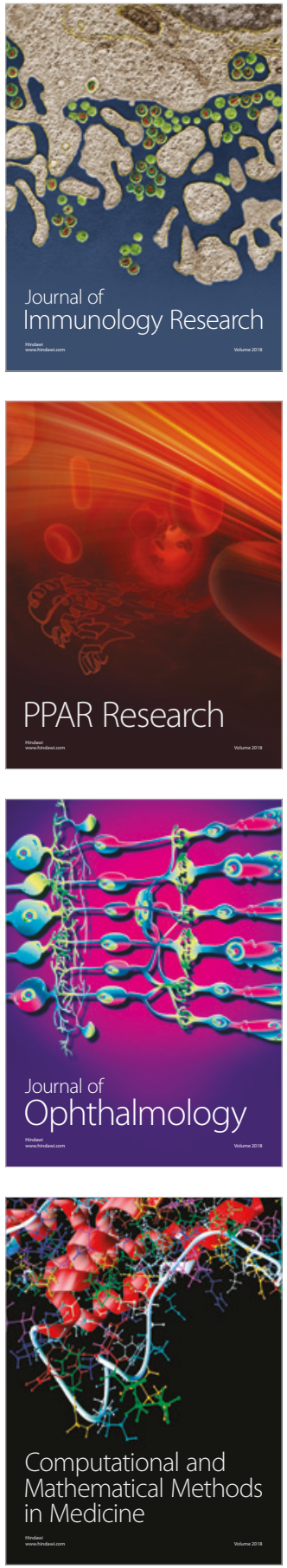

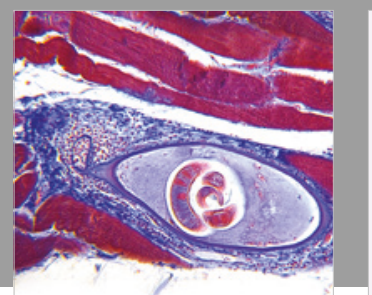

Gastroenterology Research and Practice

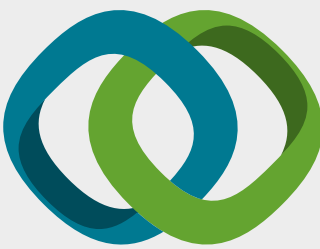

\section{Hindawi}

Submit your manuscripts at

www.hindawi.com
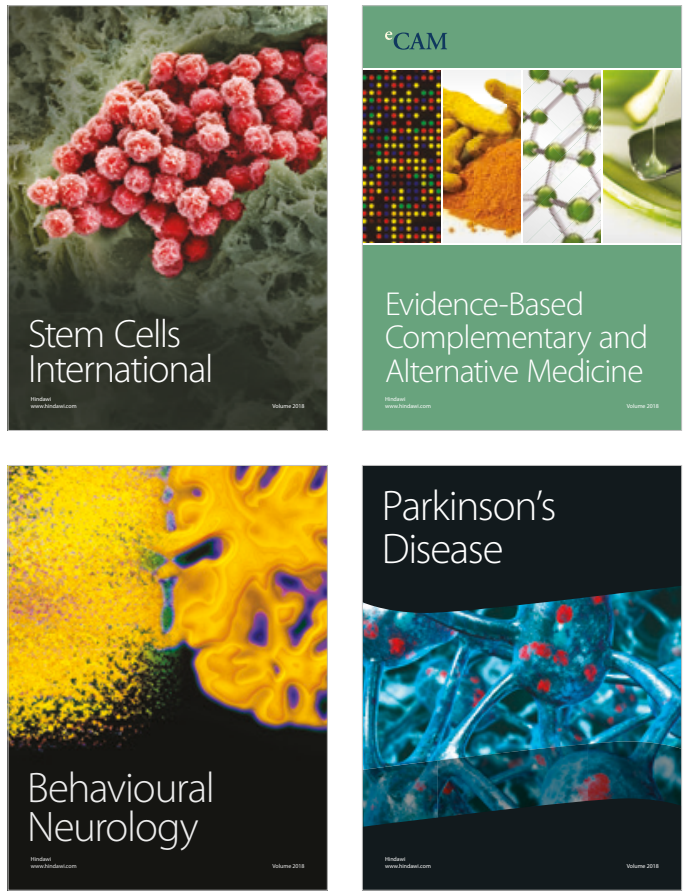

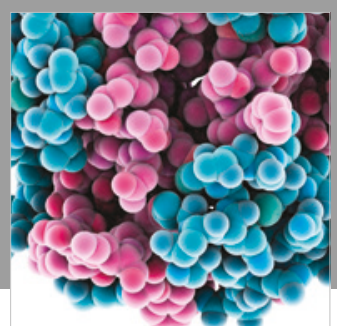

ournal of

Diabetes Research

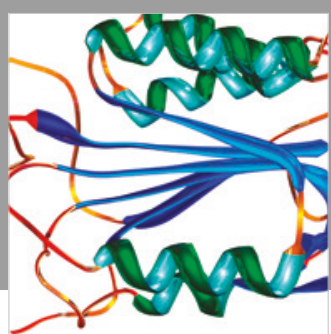

Disease Markers
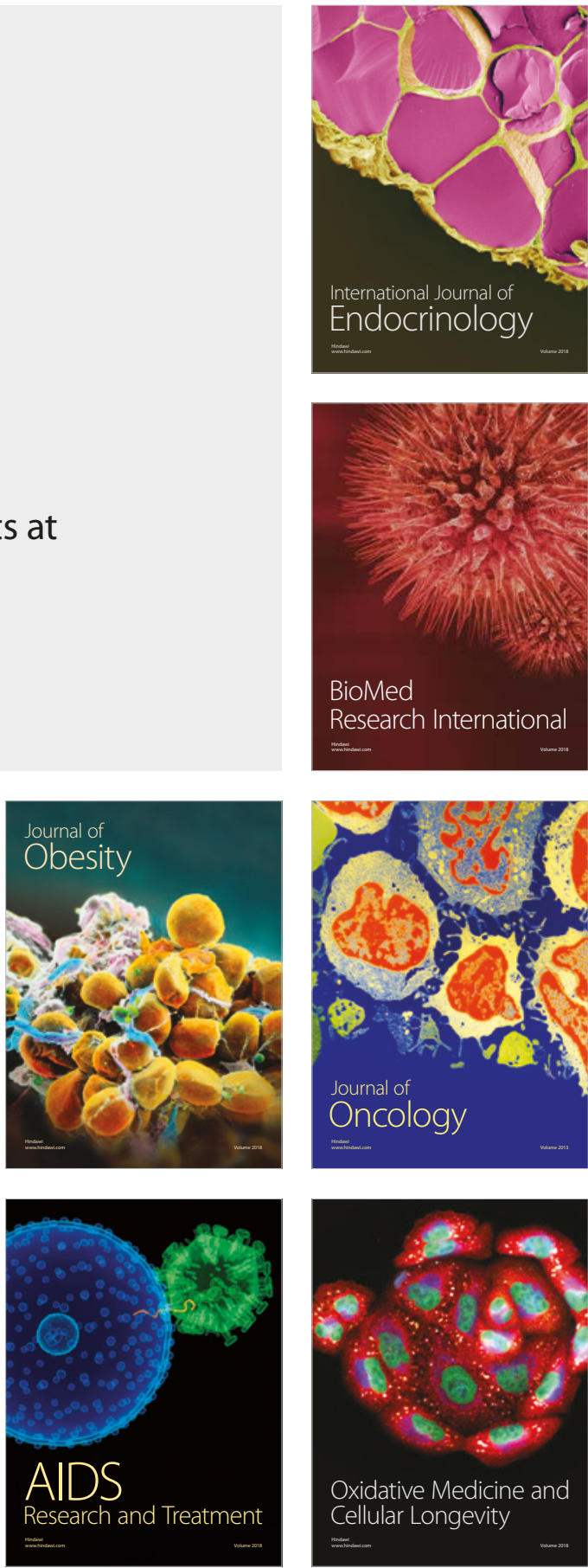